\title{
Resection of lesions in the ileum of patients with lgG4-related disease may ameliorate disease progression without steroid administration
}

\author{
Akihiro Watanabe ${ }^{1 *} \mathbb{D}$, Takashi Goto ${ }^{2}$, Hitomi Kamo², Ryuji Komine ${ }^{1}$, Naomi Kuroki², Takanobu Sugase ${ }^{2}$, \\ Tsuyoshi Takaya², Rintaro Koga², Hiroshi Hojo², Shoji Taniguchi², Kazuhiko Ibusuki and Kazumi Koga²
}

\begin{abstract}
Background: IgG4-related disease (IgG4-RD) is a pathological condition that is characterized by an infiltrate composed of IgG4-positive plasma cells and recently recognized as an immune-mediated condition. It causes tissue throughout the body to become stiff and thickened due to autoimmune reactions that cause fibrosis and scarring. Disease-related changes commonly occur in the salivary glands, bile duct, pancreas, and lungs, but are seldom seen in the small bowel. A diagnosis of IgG4-RD is suspected if a high level of IgG4 is found on a blood test. The ideal diagnostic method is pathological examination, but because the clinical manifestations of IgG4-RD are very diverse and nonspecific, the disease may often go undiagnosed until an unrelated biopsy or resection specimen is obtained. The most common treatment for IgG4-RD is steroid use. However, tapering or stopping steroid administration is seen to result in recurrence in approximately $50 \%$ of cases. A complete cure is therefore considered extremely difficult.

Case presentation: A 69-year-old man with gastrointestinal obstruction underwent small bowel resection for two lesions. On histopathological examination, the specimen showed features of IgG4-RD. We performed several tests to detect other characteristics of IgG4-RD, but were unable to find any. The patient is being followed up regularly for a year and is being observed for any symptoms of recurrence.

Conclusions: We present a case of IgG4-RD wherein the ileum wall was significantly sclerosed, leading to gastrointestinal tract obstruction; therefore, we resected two sections of the ileum. We believe that resection of IgG4-RD lesions can help avoid long-term steroid use in patients, because the surgery completely eliminates the pathological origins of the condition.
\end{abstract}

Keywords: IgG4-related sclerosing disease, Small bowel resection, Steroid

\section{Background}

IgG4-related disease (IgG4-RD) is a pathological condition that is characterized by an infiltrate composed of IgG4-positive plasma cells and recently recognized as an immune-mediated condition that can affect almost any organ [1]. Usually, we suspect that it is present if a high level of IgG4 is found on a blood test. The primary method

\footnotetext{
* Correspondence: akhee1090@yahoo.co.jp

'Department of Gastrointestinal and General Surgery, Mitsui Memorial Hospital, Tokyo, Japan

Full list of author information is available at the end of the article
}

of diagnosis is pathological examination. However, the clinical manifestations of IgG4-RD are diverse and nonspecific; therefore, the disease may remain undiagnosed until an incidental biopsy or resection specimen is obtained [2].Very little is known regarding the mechanisms and processes involved in the development of this condition; however, type $2 \mathrm{~T}$-helper cells (Th2), which regulate T cell cytokines and B cell activating factor, have been suggested to be associated with the development of this disease [3].

We present a case of IgG4-RD wherein the ileum wall was significantly sclerosed, leading to gastrointestinal 
tract obstruction, due to which we resected two sections of the ileum.

\section{Case presentation}

The patient was a 69-year-old man who was a known case of type 2 diabetes mellitus (T2DM). He initially presented with sigmoid colon cancer that

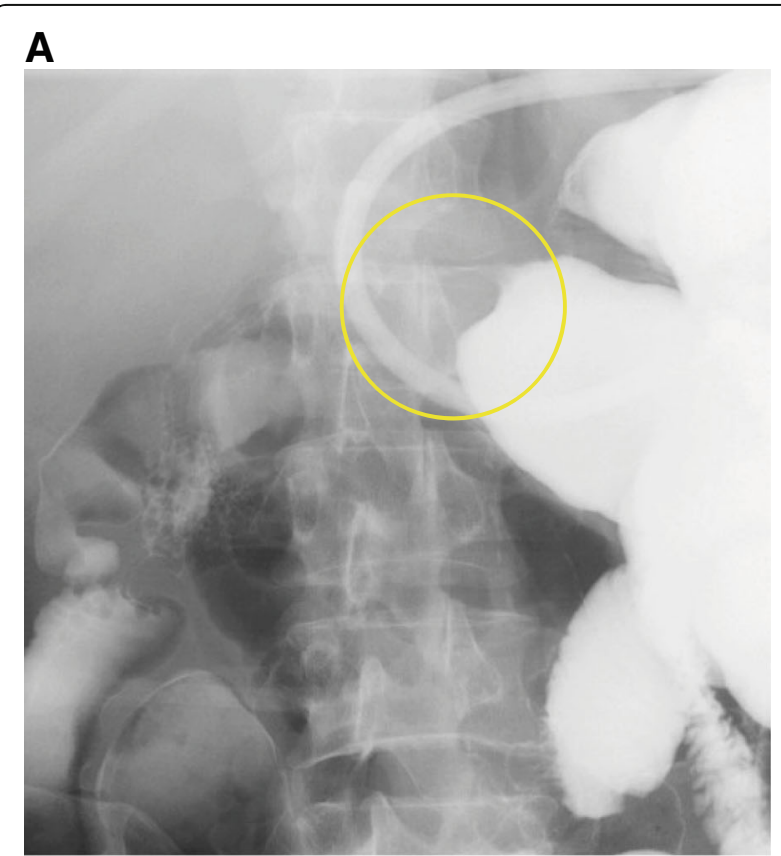

\section{B}

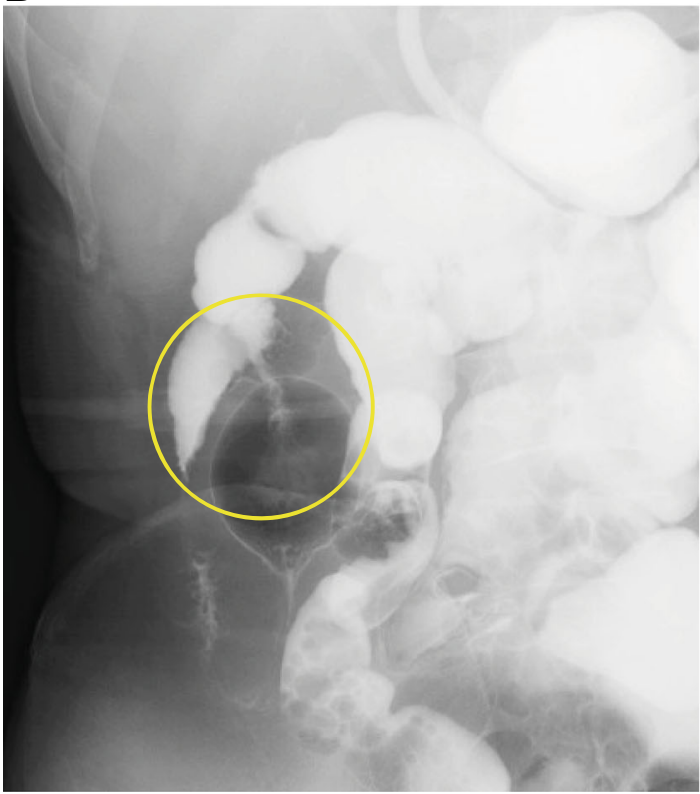

Fig. 1 We introduced $200 \mathrm{~mL}$ gastrografin into the gastrointestinal tract via a gastric tube. This examination revealed two stenosing sections of the small bowel, as seen on radiological enteroclysis (yellow circles in the image $\mathbf{a}$ and $\mathbf{b}$ ) progressed to obstructed necrotizing enteritis. He had undergone subtotal colectomy (from the ascending colon to the sigmoid colon) in our department. We had created a stoma at the ascending colon and had closed it 1 year later. During this phase, the patient's recovery was hampered by nontuberculous mycobacterial infection, for which he underwent treatment with isoniazid, rifampicin, and ethambutol at another hospital; he recovered completely from the infection.

He subsequently presented with repeated episodes of gastrointestinal tract obstruction, even after two surgeries. He was then admitted in our department to receive conservative treatment.

Two years after the first surgery, his central venous port was infected, for which he underwent treatment with antibiotics (sulbactam/ampicillin, vancomycin) at another hospital. Subsequently, he presented with obstruction of the gastrointestinal tract again. We therefore attempted to try and resolve this problem.

Blood tests showed renal dysfunction (serum creatinine level $1.18 \mathrm{mg} / \mathrm{dL}$ ), anemia (hemoglobin 9.0 $\mathrm{g} / \mathrm{dL})$, and a slightly elevated CA19-9 level $(46 \mathrm{U} / \mathrm{mL})$. Radiological enteroclysis revealed two stenosed sections of the small bowel (Fig. 1). Computed tomography of the abdomen also indicated that the wall of the small bowel was thick and stenosed (Fig. 2).

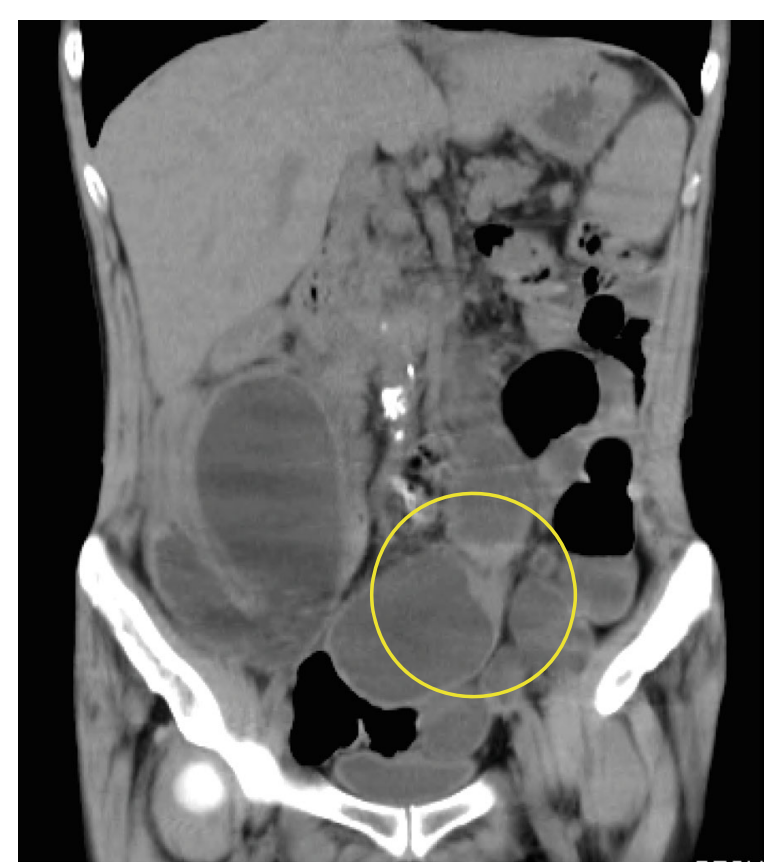

Fig. 2 The wall of the small bowel is thick and stenosed, as seen on computed tomography (yellow circle) 

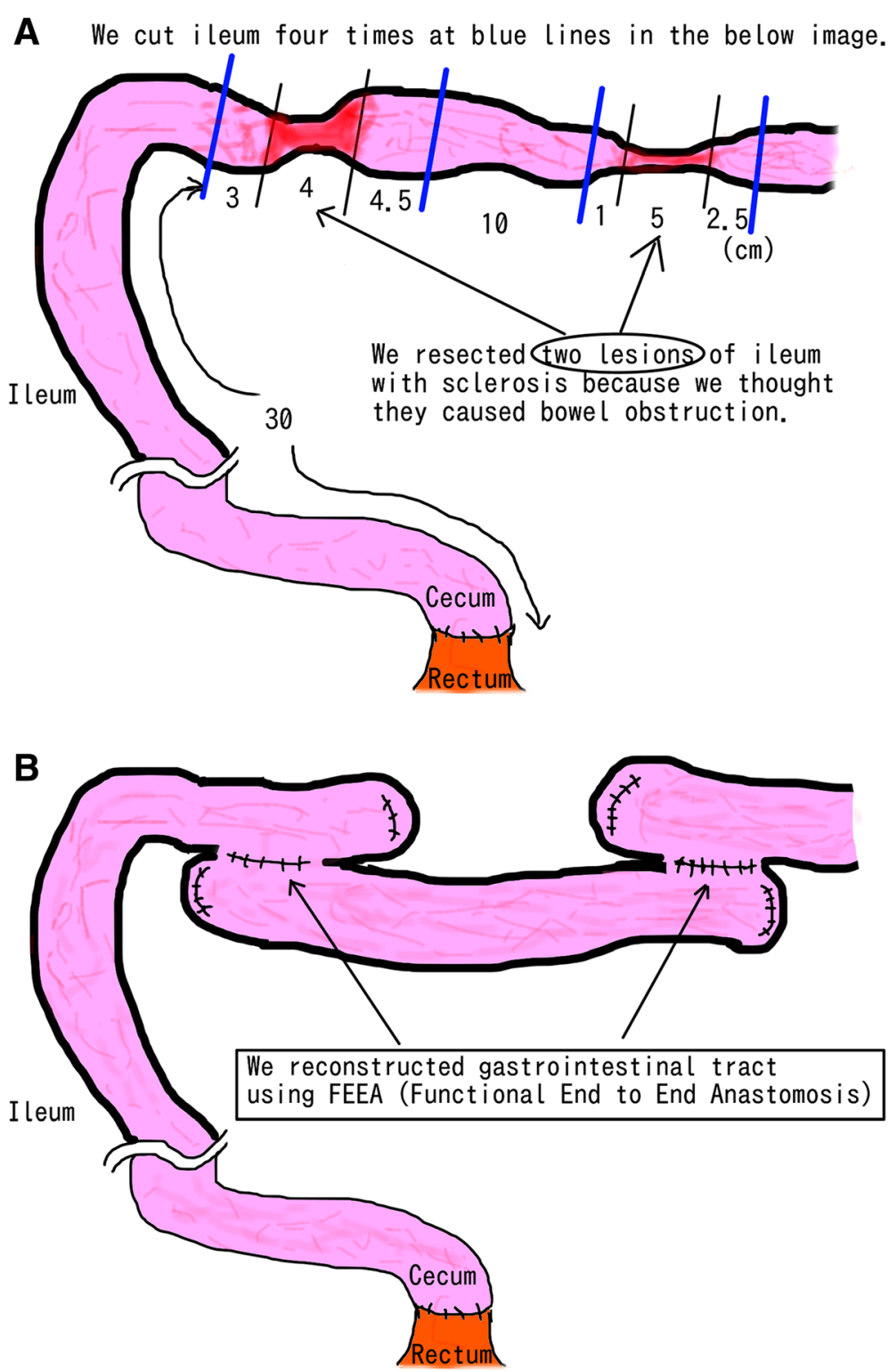

Fig. 3 The patient's cecum and rectum were anastomosed due to the previous surgery (subtotal colectomy). We recognized two sections of small bowel $33 \mathrm{~cm}$ and $53 \mathrm{~cm}$ from the terminal ileum seemed to be sclerotic, with extraordinarily thick walls (a). We resected these two lesions and anastomosed them using FEEA (functional end-to-end anastomosis), thus obtaining two specimens (b)

We continued conservative treatment (using an ileus tube), but his condition did not improve. We then decided to operate to resolve this condition.

In the surgery, we recognized two sections of small bowel $30 \mathrm{~cm}$ and $53 \mathrm{~cm}$ from the terminal ileum seemed to be fibrosed, with extraordinarily thick walls. We resected these sections with a few centimeters margin and anastomosed the remaining bowel using functional end-to-end anastomosis, finally obtaining two specimens (Figs. 3 and 4). We also performed tube splinting of the small bowel to prevent further gastrointestinal tract obstruction.
After the surgery, pathological examination yielded a large number of plasma cells and IgG4-positive cells in the specimen; the ratio of IgG4-positive cells to non-IgGpositive cells was $87 \%(350 / 404)$. We then realized that the specimen showed features of IgG4-RD (Fig. 5). Based on the diagnostic criteria, this case was classified into the probable diagnosis group.

We then conducted some tests to further clarify the diagnosis of IgG4-related disease. The blood IgG4 level was found to be $52.0 \mathrm{mg} / \mathrm{dL}$, which is within the normal limit. We performed magnetic resonance cholangiopancreatography (MRCP) to detect signs of 

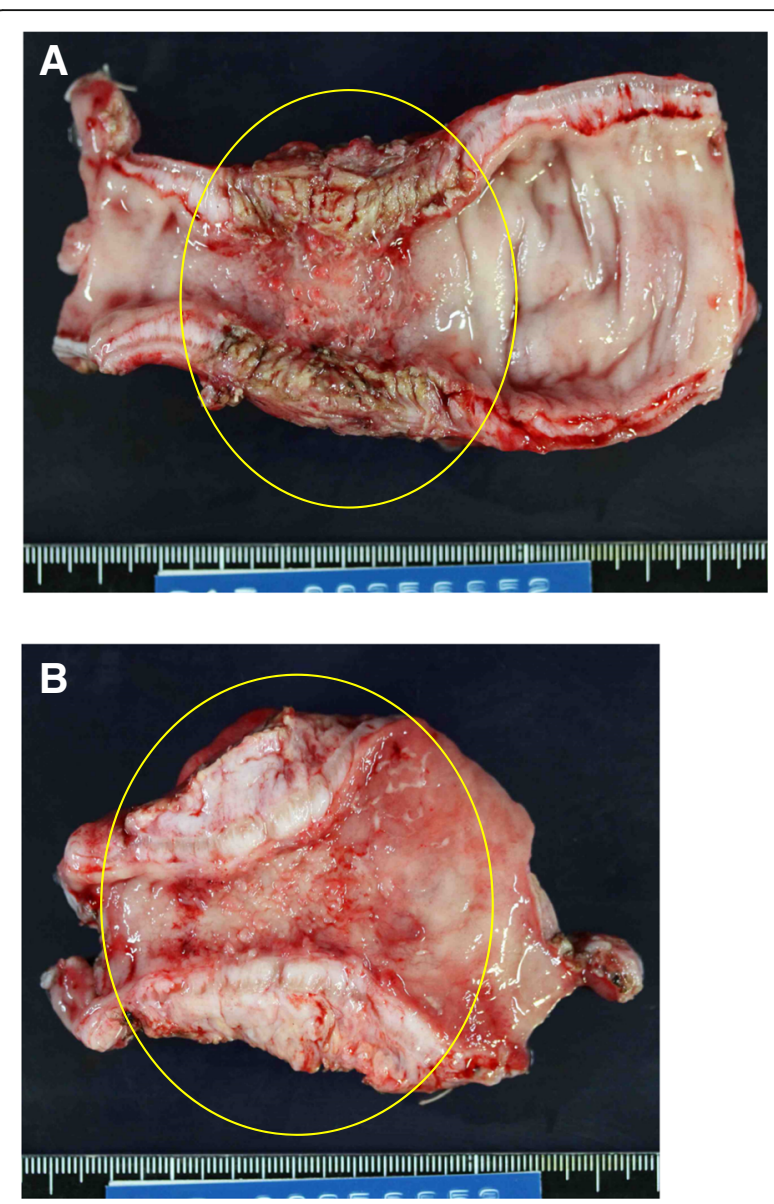

Fig. 4 Images $a$ and $b$ are macroscopic findings of the specimen. Two specimens of the ileum with a rubbery appearance and extraordinarily thick walls (yellow circles in the image $\mathbf{a}$ and $\mathbf{b}$ )

IgG4-RD in the pancreas and bile duct, but did not find any. We therefore concluded that there were no findings that suggested IgG4-RD, except for in the small bowel.

The patient was discharged to his home on the 39th postoperative day. He has been following up regularly for a year with us and has not shown any symptom of recurrence of IgG4-RD.

\section{Discussion}

The primary pathological features of IgG4-RD are swelling around the internal organs, thickened lesions due to infiltration by lymphocytes and IgG4-positive plasma cells, and fibrosis of tissue throughout the body [4].

IgG4-RD rarely affects the small bowel. Several studies describe the common primary locations of the lesions as the salivary glands, bile duct, pancreas, and lung; however, none of these studies describe any cases with lesions in the small bowel [5].

The diagnostic criteria of IgG4-RD are quite clearly defined [6]. There are three main items in the list. Patients
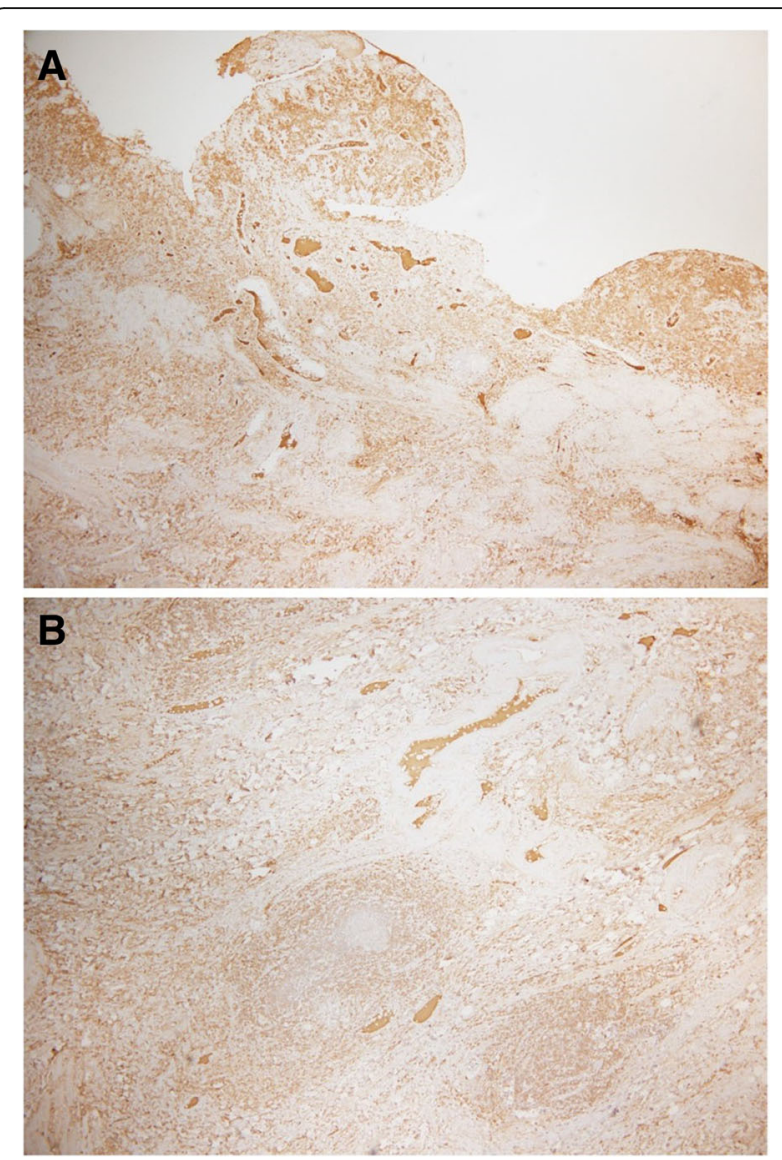

Fig. $\mathbf{5}$ Images $\mathbf{a}$ and $\mathbf{b}$ are histological findings of the specimen. A large number of plasma cells and lgG4-positive cells are seen in the specimen. The ratio of IgG4-positive cells to non lgG-positive cells is $87 \%$

who fulfill all the criteria are classified into the definite diagnostic group, while patients who fulfill criteria 1 and 3 are classified into the probable diagnostic group. The criteria are:

1. Characteristic diffuse or segmental swollen, massive, nodule, or hypertrophic lesions in single or multiple organs

2. Elevated serum IgG4 levels (above $135 \mathrm{mg} / \mathrm{dL}$ )

3. Overwhelming infiltration of lymphocytes and plasma cells and sclerosing and infiltration of IgG4-positive plasma cells; the ratio of IgG4/IgG is over $40 \%$, with over 10 IgG4-positive plasma cells seen per HPF.

The etiology of IgG4-RD seems to be autoimmune, based on the presence of high serum IgG4 levels and infiltration of IgG4-positive plasma cells. The disease is associated with both Th2 and regulatory $\mathrm{T}$ cell (Treg) immune responses. Th2 responses are also linked to the development of allergies and bronchial asthma, both of which are conditions that are more prevalent in patients with IgG4-RD [2], but the detailed etiopathology is still unknown [7]. 
The most commonly used and suitable medication used to treat IgG4-RD is steroids [8,9]. Kamisawa et al. reported that oral prednisolone is usually started at $0.6 \mathrm{mg} / \mathrm{kg} /$ day; for the maintenance, the steroid dose is then tapered by $5 \mathrm{mg}$ every 1 to 2 weeks [10]. The ideal time frame of prednisolone administration is 1 to 2 years, which can be paused in case of remission. However, the treatment in case of recurrence has not been established. Steroids are effective in treating nearly $100 \%$ of cases, but approximately $50 \%$ cases are seen to relapse if steroids are reduced or stopped. Therefore, a total cure should not be expected $[9,11,12]$.

This is a very unusual case of IgG4-RD wherein the patient presentation was different, and the signs of the condition were not present in any of the usual locations. Additionally, symptoms of recurrence have not developed even a year after surgery. In this case, the serum IgG4 level was found to be within normal limits. However, we believe that this was only because we checked the IgG4 levels after surgery. This possibly occurred because we resected the lesions that were likely producing the IgG4-positive plasma cells. This suggests that resection of the primary lesion in cases of IgG4-RD decreases the IgG4 level. This hypothesis is also supported by a report by Fujita et al., of a case of IgG4-RD treated using steroids. In this case, prednisolone was used, but resection was not performed. The patient's symptoms improved, but the number of IgG4-positive cells did not change after remission [13].

In our case, we have determined that if symptoms of recurrence are noted, the best course to follow would be steroid treatment with careful monitoring, keeping in mind the patient's T2DM and latent nontuberculous mycobacterial infection, because the utility of re-operation is yet unknown.

This patient underwent subtotal colectomy for obstructed necrotizing enteritis by sigmoid colon cancer, but it is not related to IgG4-RD. This patient developed gastrointestinal obstruction only because of sclerosis and thickening of the ileum by etiology of IgG4-RD. Resecting the lesion seemed to solve the problem, but recurrence is possible in the future. Therefore, his condition needs to be strictly monitored during follow-ups.

We suggest that operation is one of the feasible tactics of treatment of IgG4-RD in a particular situation, such as in a case we are reluctant to use steroid (e.g., a patient with diabetes mellitus). Xiao et al. reported that among 39 cases of IgG4-related cholangitis, 4 underwent surgery [13]. Among these 4 cases, 2 underwent pancreatoduodenectomy, 1 underwent choledochojejunostomy, and the fourth one underwent choledochoplasty. Over a follow-up period of 9 to 36 months, all patients achieved resolution of stricture of the bile duct and normalization of liver enzyme levels.
Other authors reported cases underwent surgery in several areas for example mesentery and lung for IgG4-RD patients and did not recur within follow-up period [14, 15]. These reports suggest that surgical intervention could be more effective than using steroids to control IgG4-RD.

\section{Conclusion}

Experience of additional cases is needed to determine the symptoms and characteristics of IgG4-RD with sclerosing changes in the small bowel, as very few cases have been reported so far. Resection of the lesions of IgG4-RD could be considered to avoid long-term steroid use in patients.

\section{Abbreviations \\ IgG4-RD: IgG4-related disease; MRCP: Magnetic resonance \\ cholangiopancreatography; T2: Type 2T-helper cell; T2DM: Type 2 \\ diabetes mellitus; Treg: Regulatory $\mathrm{T}$ cell}

Acknowledgements

We would like to thank Editage for the English language editing.

Funding

None.

\section{Authors' contributions}

TG and AW performed the operation. All authors designed and drafted the manuscript. All authors have read and approved the final version of the manuscript.

Ethics approval and consent to participate

Ethics approval and consent to publish has been obtained from the participant.

\section{Consent for publication}

Informed consent was obtained from the patient for the publication of this case report and any accompanying figures.

\section{Competing interests}

The authors declare that they have no competing interests.

\section{Publisher's Note}

Springer Nature remains neutral with regard to jurisdictional claims in published maps and institutional affiliations.

\section{Author details}

${ }^{1}$ Department of Gastrointestinal and General Surgery, Mitsui Memorial Hospital, Tokyo, Japan. '2Department of Gastrointestinal and General Surgery, Koga General Hospital, Miyazaki, Japan.

Received: 29 May 2018 Accepted: 20 November 2018 Published online: 29 December 2018

\section{References}

1. Stone JH, Zen Y, Deshpande V. IgG4-related disease. N Engl J Med. 2012; 366:539-51.

2. Weindorf SC, Frederiksen JK. IgG4-related disease: a reminder for practicing pathologists. Arch Pathol Lab Med. 2017;141(11):1476-83. https://doi.org/10. 5858/arpa.2017-0257-RA

3. Della-Torre E, Lanzillotta M, Doglioni C. Immunology of IgG4-related disease. Clin Exp Immunol. 2015;181(2):191-206. https://doi.org/10.1111/cei.12641 Epub 2015 Jun 8

4. Zen Y, Nakanuma Y. IgG4-related disease: a cross-sectional study of 114 cases. Am J Surg Pathol. 2010;34:1812-9.

5. Inoue D, Yoshida K, Yoneda N, Ozaki K, Matsubara T, Nagai K, et al. lgG4-related disease: dataset of 235 consecutive patients. Medicine (Baltimore). 2015;94:e680. https://doi.org/10.1097/MD.0000000000000680. 
6. Umehara H, Okazaki K, Masaki Y, Kawano M, Yamamoto M, Saeki T, et al. A novel clinical entity, lgG4-related disease (IgG4RD): general concept and details. Mod Rheumatol. 2012;22:1-14.

7. Yamamoto M, Takahashi H, Shinomura Y. Mechanisms and assessment of IgG4-related disease: lessons for the rheumatologist. Nat Rev Rheumatol. 2014;10:148-59. https://doi.org/10.1038/nrrheum.2013.183. Epub 2013 Dec 3.

8. Masaki Y, Sugai S, Umehara H. IgG4-related diseases including Mikulicz's disease and sclerosing pancreatitis: diagnostic insights. J Rheumatol. 2010;37:1380-5.

9. Masamune A, Nishimori I, Kikuta K, Tsuji I, Mizuno N, liyama T, et al. Randomised controlled trial of long-term maintenance corticosteroid therapy in patients with autoimmune pancreatitis. Gut. 2017;66:487-94.

10. Kamisawa T, Ryu JK, Kim MH, Okazaki K, Shimosegawa T, Chung JB. Recent advances in the diagnosis and management of autoimmune pancreatitis: similarities and differences in Japan and Korea. Gut Liver. 2013;7:394-400. https://doi.org/10.5009/gnl.2013.7.4.394. Epub 2013 Jul 11

11. Kamisawa T, Okazaki K, Kawa S, Shimosegawa T, Tanaka M, et al. Japanese consensus guidelines for management of autoimmune pancreatitis: III. Treatment and prognosis of AIP. J Gastroenterol. 2010;45:471-7. https://doi. org/10.1007/s00535-010-0221-9. Epub 2010 Mar 9.

12. Fujita K, Naganuma M, Saito E, Suzuki S, Araki A, Negi M, et al. Histologically confirmed lgG4-related small intestinal lesions diagnosed via double balloon enteroscopy. Dig Dis Sci. 2012;57:3303-6. https://doi.org/10.1007/ s10620-012-2267-4. Epub 2012 Jun 14

13. Xiao J, Xu P, Li B, Hong T, Liu W, He X, et al. Analysis of clinical characteristics and treatment of immunoglobulin G4-associated cholangitis: a retrospective cohort study of 39 IAC patients. Medicine (Baltimore). 2018; 97:e9767. https://doi.org/10.1097/MD.0000000000009767.

14. Abe A, Manabe T, Takizawa N, Ueki T, Yamada D, Nagayoshi K, Sadakari Y, Fujita H, Nagai S, Yamamoto H, Oda Y, Nakamura M. IgG4-related sclerosing mesenteritis causing bowel obstruction: a case report. Surg Case Rep. 2016; 2(1):120 Epub 2016 Oct 30

15. Okubo T, Oyamada Y, Kawada M, Kawarada Y, Kitashiro S, Okushiba S. Immunoglobulin G4-related disease presenting as a pulmonary nodule with an irregular margin. Respirol Case Rep. 2016;5(1):e00208. https://doi.org/10. 1002/rcr2.208 eCollection 2017 Jan.

\section{Submit your manuscript to a SpringerOpen ${ }^{\circ}$ journal and benefit from:}

- Convenient online submission

- Rigorous peer review

- Open access: articles freely available online

- High visibility within the field

- Retaining the copyright to your article

Submit your next manuscript at $\boldsymbol{\nabla}$ springeropen.com 